\title{
Scintigraphic texture analysis for assessment of renal allograft function
}

\author{
Ali Abbasian Ardakani ${ }^{1,2 A, B, C, D, E, F}$, Sepideh Hekmat ${ }^{3 A, B, D, E, E, G}$, Jamileh Abolghasemi ${ }^{4 C, D, E}$, Reza Reiazi ${ }^{1,2 E, F}$ \\ 'Department of Medical Physics, School of Medicine, Iran University of Medical Sciences, Tehran, Iran. \\ ${ }^{2}$ Medical Image and Signal Processing Research Core, Iran University of Medical Sciences, Tehran, Iran \\ ${ }^{3}$ Department of Nuclear Medicine, School of Medicine, Hasheminejad Hospital, Iran University of Medical Sciences, Tehran, Iran \\ ${ }^{4}$ Department of Biostatistics, School of Public Health, Iran University Medical of Sciences, Tehran, Iran
}

\section{Abstract}

Purpose: Early detection and monitoring of kidney function during the post-transplantation period is one of the most important issues for improving the accuracy of an initial diagnosis. The aim of this study was to evaluate texture analysis (TA) in scintigraphic imaging to detect changes in kidney status after transplantation.

Material and methods: Scintigraphic images were used for TA from a total of 94 kidney allografts ( 39 rejected and 55 non-rejected). Images corresponding to the frames at the $2^{\text {nd }}, 5^{\text {th }}$, and $20^{\text {th }}$ minute of the study were used to determine the optimum time point for analysis of differences in texture features between the rejected and non-rejected allografts.

Results: Linear discriminant analysis indicated the best performance at the fifth minute frame for classification of the rejected and non-rejected allografts with receiver operating characteristic curve $\left(\mathrm{A}_{\mathrm{z}}\right)$ of 0.982 , corresponding to 91.89\% sensitivity, $96.49 \%$ specificity, and $94.68 \%$ accuracy. Also, TA can differentiate acute tubular necrosis from acute rejection with $\mathrm{A}_{\mathrm{z}}$ of 0.953 corresponding to $88 \%$ sensitivity, $92.31 \%$ specificity, and $90.62 \%$ accuracy at the $5^{\text {th }}$ minute frame. The best correlation between texture feature and kidney function was achieved at the $20^{\text {th }}$ minute frame $(r=-0.396)$ for glomerular filtration rate.

Conclusions: TA has good potential for the characterisation of kidney failure after transplantation and can improve clinical diagnosis.

Key words: computer-assisted, kidney transplantation, pattern recognition system, radionuclide imaging.

\section{Introduction}

A kidney transplant is the treatment of choice for patients with chronic kidney disease (CKD) that has progressed to its final stage, i.e. end-stage renal disease (ESRD). Kidneys at ESRD are unable to remove metabolic waste, fluid, toxins, and excess fluid from the blood [1]. Despite recent improvements in treatments with immunosuppressive drugs and therapies, acute rejection (AR) causes graft loss in $12 \%$ of cases [2]. The serum creatinine ( $\mathrm{sCr}$ ) concentration remains the most commonly used endogenous filtration marker for monitoring graft function in kidney transplantation. AR manifest as a $25 \%$ or greater increase in serum creatinine level, and a decrease in urine output and glomerular filtration rate; it also causes pain and tenderness in the graft region. AR is asymptomatic in most cases and is only reflected by a rising serum creatinine level $[3,4]$. Allograft biopsy is usually performed if there is no other explanation for an increased creatinine level [5].

Although ultrasound is the chosen imaging technique for monitoring and assessing complications in a kidney allograft transplant, radionuclide imaging is advantageous compared to other imaging modalities because it provides information on functions as well as perfusion and glomer-

\section{Correspondence address:}

Sepideh Hekmat, Department of Nuclear Medicine, School of Medicine, Hasheminejad Hospital, Iran University of Medical Sciences, Tehran, Iran, phone/fax: +98 21-886-44471, e-mail: sepidhekmat@hotmail.com 
ular filtration rate (GFR). Other imaging modalities such as computed tomography (CT) and magnetic resonance imaging (MRI) provide no additional information on evaluation of renal function $[3,6]$. It seems that changes in kidney tissue are reflected in the properties of a medical image [7].

Function of a transplanted kidney has an effect on the patterns of scintigraphic images. These images consist of a range of grey-level intensities, and different types of tissue have different textures. However, computerised texture analysis (TA) has been developed to increase diagnostic confidence and relieve anxiety, and to avoid unnecessary procedures such as biopsies and laboratory tests. In conventional medical imaging, computerised TA uses a mathematical approach to detect pathological changes that are imperceptible to the human eye [8-10].

In the last decade, quantitative studies have evaluated kidney function using ultrasound [7,11-18], CT [19-21], radionuclide imaging [14,17-24], and MRI [25-28]. Previous studies have yielded promising results. The purpose of this study was to provide additional information with improved accuracy for monitoring kidney function. As mentioned before, allograft rejection causes tissue changes. These changes can affect the texture of a kidney image. The aim of this study was to evaluate TA as a non-invasive method to monitor kidney allograft transplants among follow-up patients. To the best of our knowledge, this was the first study to apply TA of radionuclide images to evaluate kidney allografts.

\section{Material and methods}

\section{Patients and image acquisition}

Data were collected from patients who had undergone kidney allograft transplantation at Hasheminejad Hospital.

All patients underwent diuretic renography according to the standard guidelines [29]. Inclusion criteria were as follows: (1) Patients had been tested for serum creatinine $(\mathrm{sCr})$ as standard care in cases with decreased allograft function or suspicion of rejection. (2) sCr level was determined on the same day as the scintigraphy examination, measured by the standard laboratory method (Jaffe) using a kinetic colorimetric assay in the central laboratory of Hasheminejad Hospital. GFR was based on serum creatinine (MDRD or CKD-EPI equations) determined as a parameter to assess kidney function [30]. (3) All patients received similar immunosuppressive therapy treatments based on steroids, mycophenolate, mofetil, and tacrolimus. (4) All patients had no previous history of transplantation.

Body mass index (BMI) was calculated as weight divided by height squared $\left(\mathrm{m}^{2}\right)$ (in kilograms per metre squared) during a physical examination. Biopsy was performed a day after scintigraphy examination.

In this study, scintigraphic images were acquired using a single-headed ADAC gamma camera equipped with a low-energy, high-resolution, parallel-hole collimator. The $10 \%$ window was centred on the ${ }^{99 \mathrm{~m}} \mathrm{Tc} 140 \mathrm{keV}$ photopeak. Following intravenous injection of $7 \mathrm{mCi}{ }^{99 \mathrm{~m}} \mathrm{Tc}$-diethylene-triaminepenta acetic acid $\left.{ }^{99 \mathrm{~m}} \mathrm{Tc}-\mathrm{DTPA}\right)$ anterior dynamic images with matrix size of $128 \times 128$ were obtained for 30 minutes.

\section{Texture feature and regions of interest selection}

Regions of Interest (ROIs) were drawn for each whole kidney. For each ROI, up to 300 texture features were extracted based on histogram, absolute gradient (spatial variation of grey-level values), run-length matrix (counts of pixel runs with the specified grey-scale value and length in a given direction), co-occurrence matrix (information about the distribution of pairs of pixels separated by given distance and direction), autoregressive model (description of correlation between neighbouring pixels), and wavelets (decomposition image frequency at different scales) $[8,9]$.

The procedure was performed on images corresponding to frames at the $2^{\text {nd }}, 5^{\text {th }}$, and $20^{\text {th }}$ minute of image acquisition. Evaluations of texture features were made from static images at intervals of the $2^{\text {nd }}, 5^{\text {th }}$, and $20^{\text {th }}$ minute (Figure 1). Scintigraphic images were considered as input in the MaZda software (version 4.6; The Technical University of Lodz, Institute of Electronics) for TA.

\section{Statistical analysis}

Data were tested for normality by the KolmogorovSmirnov test. The two-tailed Independent Samples $t$-test/ Mann-Whitney $U$-test was applied for comparisons of age, BMI, sCr, and GFR levels between the two groups (rejected and non-rejected allograft transplants). Also, the Independent Samples $t$-test/Mann-Whitney $U$-test was used to assess texture feature differences between the two groups at the $2^{\text {nd }}, 5^{\text {th }}$, and $20^{\text {th }}$ minute time frames after data acquisition had begun.

The area under the receiver operating characteristic (ROC) curve $\left(A_{z}\right)$ was calculated for each significant texture feature in order to evaluate overall performance of classification between the two groups [31]. The Pearson/Spearman correlation test was used to determine correlations between significant texture features and $\mathrm{sCr}$ level and GFR at each time frame $\left(2^{\text {nd }}, 5^{\text {th }}\right.$, and $\left.20^{\text {th }} \mathrm{min}\right)$. A $p$-value $<0.05$ was considered significant. A values were estimated beyond the 95\% confidence level. Statistical analysis was made using SPSS software version 19 (SPSS Inc., Chicago, USA).

\section{Texture analysis and classification}

Texture features that showed significant differences between two groups were used for the computerised multiparameter TA (MPTA) method. Linear discriminant analysis (LDA) was used to transform raw texture features to lower-dimensional spaces and to increase discriminative 

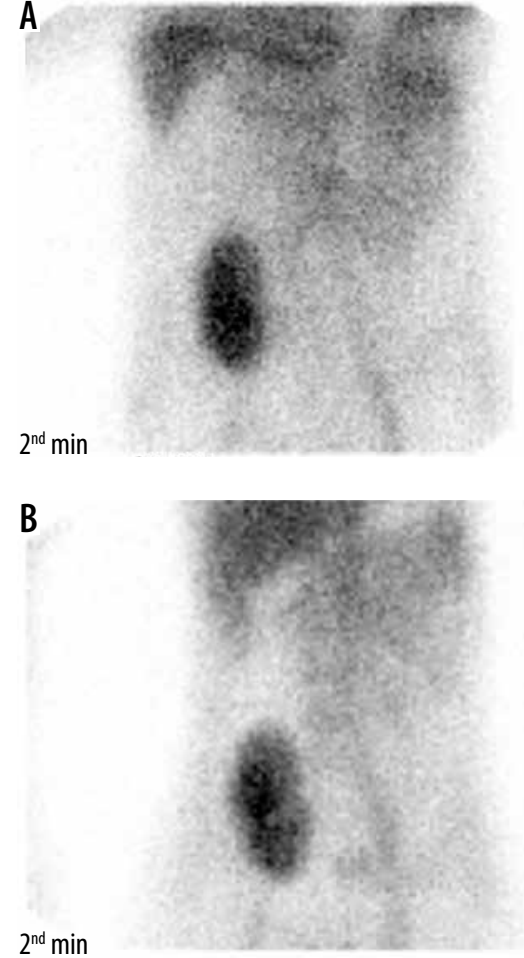
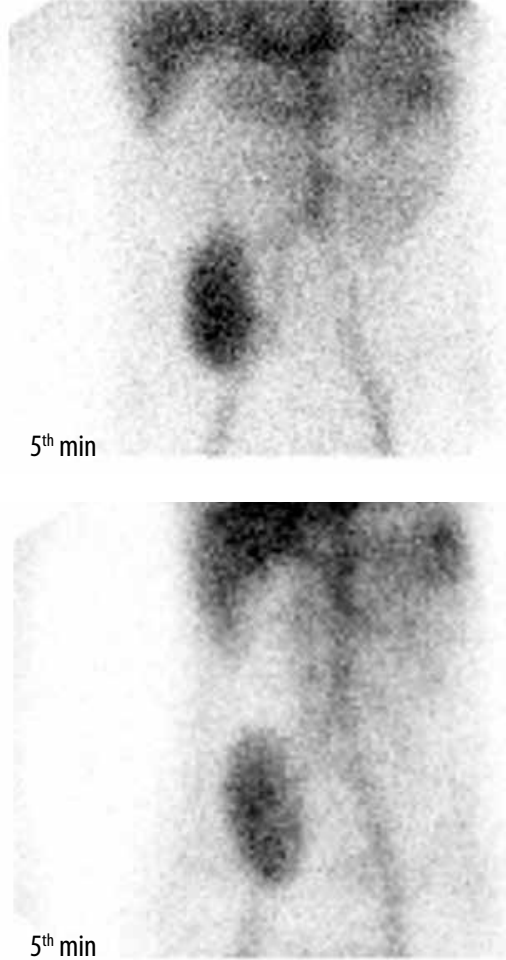

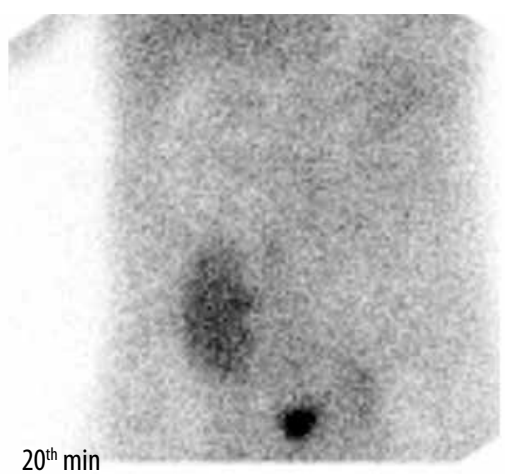

$20^{\text {th }} \min$

Figure 1. Sample of scintigraphic images of rejected (A) and non-rejected (B) kidney transplant recipients for $2^{\text {nd }}, 5^{\text {th }}$, and $20^{\text {th }}$ minute of image acquisition

power. Features processed by LDA were considered useful for pattern recognition and classification because they make data of the same class closer together and data of different classes further apart.

First nearest neighbour (1-NN) classifier was used for features determined by LDA. To compare performance of diagnostics, six well-known indexes were calculated: $A_{z}$ value, accuracy (ACC), sensitivity (SEN), specificity (SPC), positive predictive value (PPV), and negative predictive value (NPV).

The steps of the proposed computerised TA process are presented in Figure 2.

\section{Results}

\section{Demographic data of patients}

This retrospective study considered 94 biopsies of proven renal allograft recipients (50 male, 44 female). Of these 94 patients, transplanted kidneys had been rejected (AR) in 39 patients ( 25 male, 14 female). The non-rejected group consisted of 55 cases with 25 acute tubular necroses (ATN); 12 cases of obstruction and 18 normal cases. The mean duration of dialysis before transplantation was $2.5 \pm 0.6$ years. Biopsy was performed with mean $( \pm \mathrm{SD})$ of $1.37 \pm 0.97$ years after transplantation. No significant difference was determined between rejected and non-rejected grafts in terms of male and female ( $p=0.057$, Table 1$)$. Also, evaluations for difference in mean age and BMI of recipients in the rejected and non-rejected groups were determined as not statistically significant $(p=0.058$ and $p=0.092$, respectively, Table 1 ).

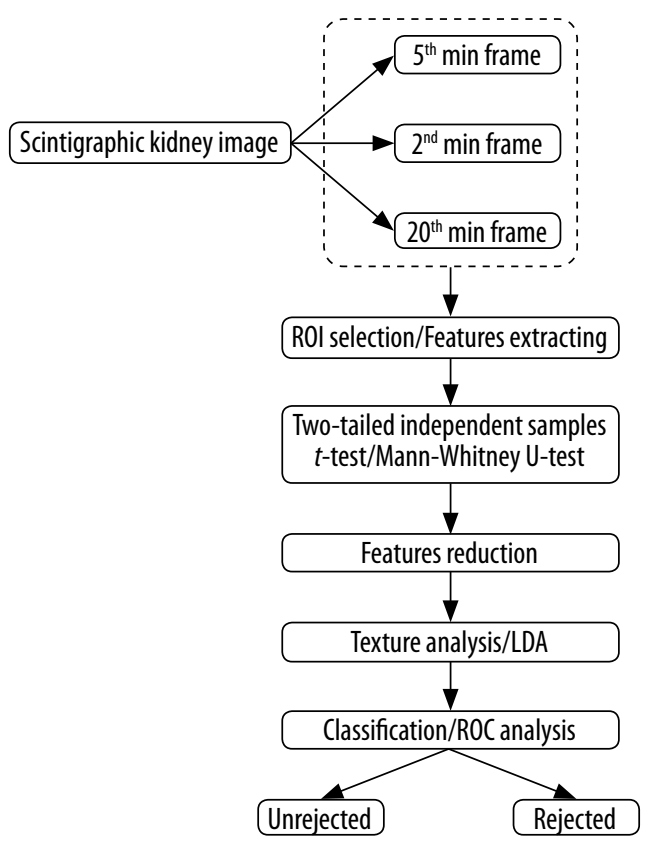

Figure 2. The computer-aided diagnosis processing steps. ROI indicates regions of interest, LDA - linear discriminant analysis, and ROC - receiver operating characteristic curve

sCr levels in patients with a rejected kidney transplant were higher than in those with non-rejected kidney transplants, and the difference was statistically significant $(p<0.001)$. GFR in patients with a non-rejected kidney transplant was higher than that in patients with rejected kidney transplants, and the difference was determined as statistically significant $(p<0.001)$. 
Table 1. Main demographic characteristics and laboratory data of rejected and non-rejected kidney transplant recipients

\begin{tabular}{|c|c|c|c|c|c|c|c|}
\hline \multirow[t]{2}{*}{ Factor } & \multicolumn{6}{|c|}{ Mean \pm SE } & \multirow{2}{*}{$\begin{array}{l}\text { Independent-sample } \\
t \text { test ( } p \text {-value) }\end{array}$} \\
\hline & \multicolumn{2}{|c|}{ Total } & \multicolumn{2}{|c|}{ Rejected } & \multicolumn{2}{|c|}{ Non-rejected } & \\
\hline Age & \multicolumn{2}{|c|}{$42.77 \pm 01.42$} & \multicolumn{2}{|c|}{$45.24 \pm 01.95$} & \multicolumn{2}{|c|}{$39.28 \pm 01.93$} & 0.058 \\
\hline Creatinine & \multicolumn{2}{|c|}{$02.73 \pm 0.13$} & \multicolumn{2}{|c|}{$03.30 \pm 0.18$} & \multicolumn{2}{|c|}{$01.92 \pm 0.11$} & $<0.001$ \\
\hline GFR & \multicolumn{2}{|c|}{$37.10 \pm 02.20$} & \multicolumn{2}{|c|}{$26.31 \pm 02.00$} & \multicolumn{2}{|c|}{$52.31 \pm 03.19$} & $<0.001$ \\
\hline \multirow[t]{2}{*}{ BMI } & \multicolumn{2}{|c|}{$23.91 \pm 0.30$} & \multicolumn{2}{|c|}{$24.34 \pm 0.40$} & \multicolumn{2}{|c|}{$23.32 \pm 0.43$} & 0.092 \\
\hline & \multicolumn{6}{|c|}{ Number (\%) } & Pearson $x^{2}$ ( $p$-value) \\
\hline \multirow[t]{2}{*}{ Gender } & Female & Male & Female & Male & Female & Male & \\
\hline & $44(46.80)$ & $50(53.20)$ & $14(35.90)$ & $25(64.10)$ & $30(54.55)$ & $25(45.45)$ & 0.057 \\
\hline
\end{tabular}

\section{Texture features and pathological changes}

The significant texture features between rejected and nonrejected kidney transplant images of the $2^{\text {nd }} \min$ frame were from the co-occurrence matrix: sum of squares $S_{(i, j)}$ and sum variance $S_{(i, j)}$; where $S_{(i, j)}$ showed the direction of the matrix construction and the inter-pixel distance $i$ along rows and $j$ along columns of the matrix, other texture features mostly belonged to wavelet: 'low-high' energy components in the sixth level wavelet decomposition (WavEnLH_s-6), WavEnLH_s-5 and 'high-low' energy components in the third level wavelet decomposition (WavEnHL_s-3); histogram: variance; and autoregressive model: Tetal and Sigma (Table 2).

The significant texture features between rejected and non-rejected kidney transplant images of frames at the $5^{\text {th }}$ min were from the co-occurrence matrix: correlate $S_{(i, j)}$, sum entropy $S_{(i, j)}$ and sum variance $S_{(i, j)}$. Other texture features mostly belonged to wavelet: WavEnLH_s-6; WavEnHH_s-6; histogram: skewness; and autoregressive model: sigma (Table 3 ).

The significant texture features between rejected and non-rejected kidney transplant images of the $20^{\text {th }}$ min frame were from the autoregressive model: Teta 2 and Teta3; histogram: percentile_1\% and percentile_10\%; runlength matrix: short run emphasis in 45-degree, and vertical direction, run length non-uniformity in 45-degree, and vertical direction and fraction of image in runs in 45-degree direction; wavelet: WavEnHH_s-5, WavEnLH_s-2, WavEnLH_s-1, WavEnLL_s-4, WavEnHL_s-2; co-occurrence matrix: inverse difference moment $\mathrm{S}_{(\mathrm{i}, \mathrm{j})}$; absolute gradient: percentage of pixels with nonzero gradient matrix and mean of absolute gradient matrix (Table 4).

\section{Correlation between texture features and serum creatinine levels}

Among all the significant texture features, some indicated a significant positive or negative correlation with sCr (or GFR). The highest correlation coefficients at the $2^{\text {nd }}$ min frame for sCr and GFR are sum variance $\mathrm{S}(2,-2)$ $(r=-0.346, p=0.001)$ and sum variance $\mathrm{S}(2,-2)(r=0.315$, $p=0.002$ ), respectively (Table 2 ). The highest correlation coefficients at the min $5^{\text {th }}$ min frame or sCr and GFR are correlate $S(0,2)(r=-0.398, p<0.001)$ and correlate $S(0,2)$ $(r=0.351, p=0.001)$, respectively (Table 3$)$, and the highest correlation coefficients at the $20^{\text {th }}$ min frame for $\mathrm{sCr}$ and GFR are Teta3 $(r=0.392, p<0.001)$ and the short run emphasis in the vertical direction $(r=-0.396, p<0.001)$, respectively (Table 4 ).

\section{Area under the roc curve for classification of rejected and non-rejected transplantation}

Roc analysis indicated that texture features of wavelet (WavEnLH_s-6) and histogram (variance) had the highest discriminatory power in terms of differentiation between rejected and non-rejected cases of kidney transplant, with $A_{z}$ values of 0.693 and 0.683 , respectively, at the 2 min frame. $A_{z}$ values of other texture features are listed in Table 2. Table 3 shows that $A_{z}$ values of the co-occurrence matrix (correlate $S(0,1)$ and correlate $S(0,2)$ ) and histogram (skewness) texture features had higher discriminatory power than others to classify rejected and non-rejected cases at the $5^{\text {th }} \min$ frame. The $A_{z}$ value of correlate $S(0,1)$, correlate $S(0,2)$, and skewness were $0.705,0.698$, and 0.694 , respectively. The $A_{z}$ values of texture features of the autoregressive model (Teta2, $A_{z}=0.674$, and Teta3, $A_{z}=0.661$ ) and histogram (percentile_1\%, $A_{z}=0.660$ ) were higher in terms of difference between rejected and non-rejected cases at the $20^{\text {th }}$ min frame. The $A_{z}$ values of each significant feature are listed in Table 4.

\section{Texture analysis and classification}

Diagnostic performance of the MPTA for classification and comparison between rejected and non-rejected cases are shown in Table 5. Figure 3A shows ROC curves of the proposed MPTA that demonstrated the best performance at the $5^{\text {th }}$ min frame in terms of classification between rejected and non-rejected cases with $\mathrm{A}_{\mathrm{z}}=0.982$, which corresponds to $91.89 \%$ sensitivity, $96.49 \%$ specificity, $94.68 \%$ accuracy, $94.44 \%$ PPV, and $94.83 \%$ NPV. Performances of other frames are listed in Table 5. 
Table 2. Pearson correlations between serum creatinine (sCr) level, GFR, and texture feature in kidney transplant recipients, $2^{\text {nd }}$ min frame after acquisition

\begin{tabular}{|c|c|c|c|c|}
\hline \multirow[t]{2}{*}{ Texture feature name } & \multicolumn{2}{|c|}{ Pearson coefficient ( $p$-value) } & \multirow{2}{*}{$\begin{array}{l}\text { Independent-sample } \\
\text { test ( } p \text {-value) }\end{array}$} & \multirow[t]{2}{*}{$A_{z}$ value* } \\
\hline & scrlevel & GFR & & \\
\hline WavEnLH_s-6 & $-0.306(0.003)$ & $0.299(0.003)$ & 0.001 & $0.693(0.585,0.800)$ \\
\hline Variance & $-0.344(0.001)$ & $0.313(0.002)$ & 0.003 & $0.683(0.571,0.794)$ \\
\hline Sum of Squares $S(1,0)$ & $-0.342(0.001)$ & $0.312(0.002)$ & 0.003 & $0.680(0.569,0.792)$ \\
\hline Sum Variance $S(1,0)$ & $-0.341(0.001)$ & $0.311(0.002)$ & 0.003 & $0.680(0.568,0.791)$ \\
\hline Sum Variance $S(2,0)$ & $-0.343(0.001)$ & $0.312(0.002)$ & 0.003 & $0.679(0.568,0.791)$ \\
\hline Sum of Squares $S(1,1)$ & $-0.338(0.001)$ & $0.308(0.003)$ & 0.003 & $0.679(0.568,0.791)$ \\
\hline$S(1,-1)$ sum of squares & $-0.344(0.001)$ & $0.315(0.002)$ & 0.003 & $0.679(0.568,0.791)$ \\
\hline Sum Variance $S(1,-1)$ & $-0.342(0.001)$ & $0.313(0.002)$ & 0.003 & $0.679(0.568,0.791)$ \\
\hline Sum of Squares $S(0,1)$ & $-0.338(0.001)$ & $0.308(0.003)$ & 0.003 & $0.679(0.567,0.791)$ \\
\hline Sum Variance $S(0,1)$ & $-0.339(0.001)$ & $0.308(0.003)$ & 0.003 & $0.679(0.567,0.790)$ \\
\hline Sum Variance $S(1,1)$ & $-0.337(0.001)$ & $0.306(0.003)$ & 0.003 & $0.678(0.566,0.790)$ \\
\hline Sum Variance $S(5,0)$ & $-0.338(0.001)$ & $0.307(0.003)$ & 0.004 & $0.677(0.565,0.789)$ \\
\hline Sum of Squares $S(2,0)$ & $-0.340(0.001)$ & $0.312(0.002)$ & 0.004 & $0.677(0.565,0.789)$ \\
\hline Sum of Squares $S(0,2)$ & $-0.338(0.001)$ & $0.309(0.002)$ & 0.004 & $0.677(0.565,0.789)$ \\
\hline Sum Variance $S(2,2)$ & $-0.334(0.001)$ & $0.302(0.003)$ & 0.004 & $0.677(0.565,0.789)$ \\
\hline Sum Variance $S(4,0)$ & $-0.338(0.001)$ & $0.307(0.003)$ & 0.004 & $0.676(0.564,0.789)$ \\
\hline Sum Variance $S(3,0)$ & $-0.339(0.001)$ & $0.308(0.003)$ & 0.004 & $0.676(0.564,0.788)$ \\
\hline Sum Variance $S(0,2)$ & $-0.339(0.001)$ & $0.307(0.003)$ & 0.004 & $0.676(0.564,0.788)$ \\
\hline Sum Variance $S(2,-2)$ & $-0.346(0.001)$ & $0.315(0.002)$ & 0.004 & $0.676(0.564,0.788)$ \\
\hline Sum Variance $S(4,4)$ & $-0.329(0.001)$ & $0.296(0.004)$ & 0.004 & $0.676(0.563,0.788)$ \\
\hline Sum of Squares $S(2,2)$ & $-0.336(0.001)$ & $0.307(0.003)$ & 0.004 & $0.675(0.563,0.787)$ \\
\hline WavEnLH_s-5 & $-0.345(0.001)$ & $0.294(0.004)$ & 0.004 & $0.674(0.564,0.783)$ \\
\hline Sum Variance $S(3,3)$ & $-0.330(0.001)$ & $0.294(0.004)$ & 0.004 & $0.674(0.562,0.786)$ \\
\hline Sum Variance $S(5,5)$ & $-0.320(0.002)$ & $0.288(0.005)$ & 0.004 & $0.674(0.561,0.787)$ \\
\hline Sum of Squares $S(2,-2)$ & $-0.340(0.001)$ & $0.302(0.003)$ & 0.004 & $0.674(0.561,0.786)$ \\
\hline sum Varnc $S(3,-3)$ & $-0.344(0.001)$ & $0.313(0.002)$ & 0.004 & $0.673(0.560,0.785)$ \\
\hline Sum of Squares $S(0,3)$ & $-0.332(0.001)$ & $0.305(0.003)$ & 0.005 & $0.672(0.560,0.785)$ \\
\hline Sum of Squares $S(3,3)$ & $-0.333(0.001)$ & $0.304(0.003)$ & 0.005 & $0.672(0.560,0.784)$ \\
\hline Sum of Squares $S(3,0)$ & $-0.332(0.001)$ & $0.305(0.003)$ & 0.005 & $0.671(0.559,0.784)$ \\
\hline Sum Variance $S(0,3)$ & $-0.340(0.001)$ & $0.309(0.002)$ & 0.005 & $0.671(0.559,0.784)$ \\
\hline Teta1 & $-0.141(0.176)$ & $0.122(0.240)$ & 0.025 & $0.667(0.550,0.785)$ \\
\hline Sigma & $0.336(0.001)$ & $-0.280(0.006)$ & 0.006 & $0.663(0.550,0.776)$ \\
\hline WavEnHL_s-3 & $-0.271(0.008)$ & $0.267(0.009)$ & 0.035 & $0.663(0.518,0.749)$ \\
\hline
\end{tabular}

* Numbers in parentheses are $95 \%$ confidence intervals.

Because $45 \%$ of non-rejected cases were ATN, MPTA was also applied to differentiate AR from ATN. In this regard, diagnostic performance of the MPTA for classification and comparison between $39 \mathrm{AR}$ and $25 \mathrm{ATN}$ cases are shown in Table 5. Figure 3B shows ROC curves of the proposed MPTA that demonstrated the best performance at the $5^{\text {th }}$ min frame in terms of classification between AR and ATN cases with $\mathrm{A}_{z}=0.953$, which corresponds to $88 \%$ sensitivity, $92.31 \%$ specificity, $90.62 \%$ accuracy, $88 \%$ PPV, and $92.31 \%$ NPV. Performances of other frames are listed in Table 5.

\section{Discussion}

The primary objective of this study was to evaluate the potential of TA as a non-invasive method to monitor function in kidney allograft transplants using scintigraphic imaging. Up to 300 texture features were extracted from 
Table 3. Pearson correlations between serum creatinine ( $\mathrm{s}(\mathrm{r})$ level, GFR, and texture feature in kidney transplant recipients, $5^{\text {th }}$ min frame after acquisition

\begin{tabular}{|c|c|c|c|c|}
\hline \multirow[t]{2}{*}{ Texture feature name } & \multicolumn{2}{|c|}{ Pearson coefficient ( $p$-value) } & \multirow{2}{*}{$\begin{array}{l}\text { Independent-sample test } \\
\text { ( } p \text {-value) }\end{array}$} & \multirow[t]{2}{*}{$A_{z}$ value* } \\
\hline & sCr level & GFR & & \\
\hline Correlate $S(0,1)$ & $-0.394(<0.001)$ & $0.349(0.001)$ & 0.001 & $0.705(0.592,0.819)$ \\
\hline Correlate $S(0,2)$ & $-0.398(<0.001)$ & $0.351(0.001)$ & 0.001 & $0.698(0.584,0.812)$ \\
\hline Skewness & $-0.231(0.025)$ & $0.206(0.030)$ & 0.001 & $0.694(0.588,0.800)$ \\
\hline Correlate $S(1,1)$ & $-0.317(0.002)$ & $0.279(0.007)$ & 0.010 & $0.693(0.578,0.809)$ \\
\hline Correlate $S(1,0)$ & $-0.313(0.002)$ & $0.279(0.006)$ & 0.002 & $0.693(0.578,0.808)$ \\
\hline Correlate $S(0,3)$ & $-0.397(<0.001)$ & $0.350(0.001)$ & 0.002 & $0.691(0.576,0.806)$ \\
\hline Correlate $S(1,-1)$ & $-0.394(<0.001)$ & $0.252(0.001)$ & 0.002 & $0.688(0.574,0.801)$ \\
\hline Sigma & $0.343(0.001)$ & $0.291(0.004)$ & 0.002 & $0.688(0.573,0.802)$ \\
\hline Correlate $S(2,-2)$ & $-0.381(<0.001)$ & $0.342(0.001)$ & 0.016 & $0.683(0.566,0.800)$ \\
\hline Correlate $S(2,2)$ & $-0.305(0.003)$ & $0.267(0.009)$ & 0.016 & $0.683(0.566,0.800)$ \\
\hline Correlate $S(2,0)$ & $-0.309(0.002)$ & $0.274(0.008)$ & 0.022 & $0.681(0.565,0.797)$ \\
\hline Correlate $S(0,4)$ & $-0.384(<0.001)$ & $0.338(0.001)$ & 0.030 & $0.678(0.561,0.795)$ \\
\hline Correlate $S(3,0)$ & $-0.301(0.003)$ & $0.269(0.009)$ & 0.026 & $0.673(0.557,0.790)$ \\
\hline Correlate $S(2,-2)$ & $-0.381(<0.001)$ & $0.342(0.001)$ & 0.005 & $0.670(0.556,0.785)$ \\
\hline Correlate $S(3,3)$ & $-0.282(0.006)$ & $0.246(0.017)$ & 0.022 & $0.670(0.551,0.789)$ \\
\hline Correlate $S(0,5)$ & $-0.369(<0.001)$ & $0.234(0.001)$ & 0.008 & $0.662(0.544,0.780)$ \\
\hline Correlate $S(4,0)$ & $-0.288(0.005)$ & $0.258(0.012)$ & 0.028 & $0.659(0.542,0.776)$ \\
\hline Correlate $S(5,0)$ & $-0.265(0.010)$ & $0.243(0.018)$ & 0.030 & $0.655(0.539,0.771)$ \\
\hline WavEnLH_s-6 & $\ldots(0.108)$ & $\ldots .(0.099)$ & 0.011 & $0.654(0.544,0.763)$ \\
\hline Correlate S(4,4) & $-0.243(0.018)$ & $0.212(0.040)$ & 0.032 & $0.649(0.530,0.768)$ \\
\hline Correlate $S(3,-3)$ & $-0.352(<0.001)$ & $0.324(0.001)$ & 0.032 & $0.645(0.530,0.760)$ \\
\hline Sum Variance $S(3,3)$ & $\ldots .(0.499)$ & $\ldots .(0.431)$ & 0.035 & $0.637(0.521,0.753)$ \\
\hline Sum Entropy S(3,3) & $\ldots .(0.441)$ & $\ldots .(0.323)$ & 0.047 & $0.635(0.520,0.751)$ \\
\hline Sum Variance $S(2,2)$ & $\ldots .(0.557)$ & $\ldots .(0.475)$ & 0.035 & $0.635(0.519,0.751)$ \\
\hline Sum Variance $S(4,4)$ & $\ldots .(0.499)$ & $\ldots .(0.428)$ & 0.038 & $0.634(0.518,0.751)$ \\
\hline Sum Variance $S(0,2)$ & $\ldots(0.557)$ & $\ldots(0.462)$ & 0.038 & $0.634(0.518,0.750)$ \\
\hline Sum Variance $S(1,1)$ & $\ldots .(0.629)$ & $\ldots .(0.523)$ & 0.037 & $0.634(0.518,0.749)$ \\
\hline WavEnHH_s-6 & $\ldots .(0.495)$ & $\ldots .(0.536)$ & 0.029 & $0.633(0.521,0.745)$ \\
\hline Sum Variance $S(0,3)$ & $\ldots .(0.527)$ & $\ldots .(0.436)$ & 0.037 & $0.633(0.517,749)$ \\
\hline Sum Entropy S(2,2) & $\ldots .(0.511)$ & $\ldots(0.367)$ & 0.044 & $0.632(0.517,0.748)$ \\
\hline Sum Variance $S(0,1)$ & $\ldots . .(0.625)$ & $\ldots .(0.509)$ & 0.038 & $0.632(0.516,0.748)$ \\
\hline Sum Variance $S(3,-3)$ & $\ldots .(0.459)$ & $\ldots .(0.378)$ & 0.036 & $0.632(0.516,0.748)$ \\
\hline Sum Entropy $S(0,3)$ & $\ldots .(0.521)$ & $\ldots .(0.383)$ & 0.046 & $0.632(0.516,0.747)$ \\
\hline Sum Variance $S(0,4)$ & $\ldots .(0.488)$ & $\ldots .(0.421)$ & 0.038 & $0.632(0.515,0.748)$ \\
\hline Sum Entropy $S(1,1)$ & $\ldots .(0.555)$ & $\ldots .(0.390)$ & 0.041 & $0.631(0.516,0.747)$ \\
\hline Sum Variance $S(3,0)$ & $\ldots(0.548)$ & $\ldots .(0.441)$ & 0.038 & $0.631(0.515,0.748)$ \\
\hline Sum Variance $S(0,5)$ & $\ldots(0.458)$ & $\ldots .(0.397)$ & 0.042 & $0.631(0.515,0.748)$ \\
\hline Sum Variance $S(5,5)$ & $\ldots(0.553)$ & $\ldots(0.460)$ & 0.045 & $0.631(0.515,0.748)$ \\
\hline Sum Variance $S(2,-2)$ & $\ldots .(0.516)$ & $\ldots .(0.424)$ & 0.037 & $0.631(0.515,0.747)$ \\
\hline Sum Entropy $S(0,4)$ & $\ldots(0.486)$ & $\ldots .(0.365)$ & 0.046 & $0.630(0.514,0.747)$ \\
\hline
\end{tabular}

* Numbers in parentheses are $95 \%$ confidence intervals. 
Table 4. Pearson correlations between serum creatinine ( $s(r)$ level, GFR, and texture feature in kidney transplant recipients, $20^{\text {th }}$ min frame after acquisition

\begin{tabular}{|c|c|c|c|c|}
\hline \multirow[t]{2}{*}{ Texture feature name } & \multicolumn{2}{|c|}{ Pearson coefficient ( $p$-value) } & \multirow{2}{*}{$\begin{array}{l}\text { Independent-sample test } \\
\text { ( } p \text {-value) }\end{array}$} & \multirow[t]{2}{*}{$A_{z}$ value * } \\
\hline & sCr level & GFR & & \\
\hline Teta2 & $-0.347(0.001)$ & $0.337(0.001)$ & 0.007 & $0.674(0.562,0.786)$ \\
\hline Teta3 & $0.392(<0.001)$ & $-0.337(0.001)$ & 0.006 & $0.661(0.548,0.773)$ \\
\hline Percentile_1\% & $-0.202(0.051)$ & $0.228(0.027)$ & 0.005 & $0.660(0.545,0.776)$ \\
\hline Short Run Emphasis in 45-Degree Direction & $0.342(0.001)$ & $-0.358(<0.001)$ & 0.012 & $0.657(0.543,0.770)$ \\
\hline Percentile_10\% & $-0.264(0.010)$ & $0.292(0.004)$ & 0.005 & $0.653(0.538,0.769)$ \\
\hline Inverse Difference Moment $S(1,1)$ & $-0.363(<0.001)$ & $0.367(<0.001)$ & 0.007 & $0.653(0.538,0.769)$ \\
\hline Inverse Difference Moment S(2,2) & $-0.349(0.001)$ & $0.346(0.001)$ & 0.011 & $0.650(0.534,0.766)$ \\
\hline WavEnHH_s-5 & $0.044(0.670)$ & $-0.299(0.003)$ & 0.014 & $0.649(0.537,0.761)$ \\
\hline $\begin{array}{l}\text { Run Length Non-Uniformity in 45-Degree } \\
\text { Direction }\end{array}$ & $0.167(0.108)$ & $-0.108(0.300)$ & 0.007 & $0.648(0.537,0.758)$ \\
\hline $\begin{array}{l}\text { Run Length Non-Uniformity in Vertical } \\
\text { Direction }\end{array}$ & $0.237(0.022)$ & $-0.187(0.017)$ & 0.012 & $0.648(0.536,0.761)$ \\
\hline WavEnLH_s-2 & $0.364(<0.001)$ & $-0.378(<0.001)$ & 0.017 & $0.648(0.533,0.762)$ \\
\hline WavEnLH_s-1 & $0.366(<0.001)$ & $-0.376(<0.001)$ & 0.023 & $0.647(0.532,0.762)$ \\
\hline Short Run Emphasis in Vertical Direction & $0.376(<0.001)$ & $-0.396(<0.001)$ & 0.020 & $0.647(0.532,0.762)$ \\
\hline Mean of Absolute Gradient Matrix & $0.349(0.001)$ & $-0.360(<0.001)$ & 0.008 & $0.646(0.532,0.760)$ \\
\hline $\begin{array}{l}\text { Fraction of Image in Runs in 45-Degree } \\
\text { Direction }\end{array}$ & $0.366(<0.001)$ & $-0.354(<0.001)$ & 0.014 & $0.646(0.529,0.762)$ \\
\hline Inverse Difference Moment S(3,3) & $-0.327(0.001)$ & $0.325(0.001)$ & 0.016 & $0.645(0.528,0.762)$ \\
\hline WavEnLL_s-4 & $-0.295(0.004)$ & $0.320(0.002)$ & 0.006 & $0.644(0.532,0.757)$ \\
\hline WavEnHL_s-2 & $0.341(0.001)$ & $-0.348(0.001)$ & 0.005 & $0.644(0.531,0.757)$ \\
\hline $\begin{array}{l}\text { Percentage of Pixels with Nonzero } \\
\text { Gradient Matrix }\end{array}$ & $0.365(<0.001)$ & $-0.372(<0.001)$ & 0.008 & $0.644(0.529,0.760)$ \\
\hline
\end{tabular}

*Numbers in parentheses are $95 \%$ confidence intervals.

Table 5. Diagnostic performance of proposed multi-parameter texture analysis for classification of rejected and non-rejected in kidney transplant recipients

\begin{tabular}{|c|c|c|c|c|c|c|c|c|c|c|}
\hline Groups & $\begin{array}{c}\text { Time } \\
\text { intervals }\end{array}$ & $\begin{array}{l}\text { Method of } \\
\text { texture analysis }\end{array}$ & $\begin{array}{l}\text { SEN } \\
(\%)\end{array}$ & $\begin{array}{l}\text { SPC } \\
(\%)\end{array}$ & $\begin{array}{l}\text { ACC } \\
(\%)\end{array}$ & $\begin{array}{l}\text { PPV } \\
(\%)\end{array}$ & $\begin{array}{l}\text { NPV } \\
(\%)\end{array}$ & $A_{z}$ value* & $\begin{array}{c}\text { Asymptotic } \\
\text { significance, } p^{* *}\end{array}$ & $\begin{array}{c}\text { Correct } \\
\text { classification }\end{array}$ \\
\hline \multirow[t]{3}{*}{ R vs. NR } & $2 \min$ & LDA & 87.18 & 89.10 & 88.30 & 85 & 90.74 & $\begin{array}{c}0.932 \\
(0.885,0.980)\end{array}$ & $<0.001$ & $\begin{array}{c}83 / 94 \\
(88.30 \%)\end{array}$ \\
\hline & $5 \mathrm{~min}$ & LDA & 92.30 & 96.36 & 94.68 & 94.74 & 94.64 & $\begin{array}{c}0.982 \\
(0.963,1.000)\end{array}$ & $<0.001$ & $\begin{array}{c}89 / 94 \\
(94.68 \%)\end{array}$ \\
\hline & $20 \mathrm{~min}$ & LDA & 76.92 & 83.64 & 80.85 & 76.92 & 83.64 & $\begin{array}{c}0.847 \\
(0.772,0.923)\end{array}$ & $<0.001$ & $\begin{array}{c}76 / 94 \\
(80.85 \%)\end{array}$ \\
\hline \multirow[t]{3}{*}{ ATN vs. AR } & $2 \min$ & LDA & 76.00 & 87.18 & 82.81 & 79.17 & 85.00 & $\begin{array}{c}0.898 \\
(0.823,0.974)\end{array}$ & $<0.001$ & $\begin{array}{c}53 / 64 \\
(82.81 \%)\end{array}$ \\
\hline & $5 \mathrm{~min}$ & LDA & 88.00 & 92.31 & 90.62 & 88.00 & 92.31 & $\begin{array}{c}0.953 \\
(0.907,0.999)\end{array}$ & $<0.001$ & $\begin{array}{c}58 / 64 \\
(90.62 \%)\end{array}$ \\
\hline & $20 \mathrm{~min}$ & LDA & 76.00 & 74.36 & 75.00 & 65.52 & 82.85 & $\begin{array}{c}0.804 \\
(0.693,0.915)\end{array}$ & $<0.001$ & $\begin{array}{c}48 / 64 \\
(75.00 \%)\end{array}$ \\
\hline
\end{tabular}

SEN - sensitivity, SPC - specificity, ACC - accuracy, PPV - positive predictive value, NPV - negative predictive value, - area under ROC curve, R - rejected, NR - non-rejected, ATN - acute tubular necrosis, AR - acute rejection

*Numbers in parentheses are $95 \%$ confidence intervals

${ }^{* *}$ Null hypothesis: true area $=0.5$. 

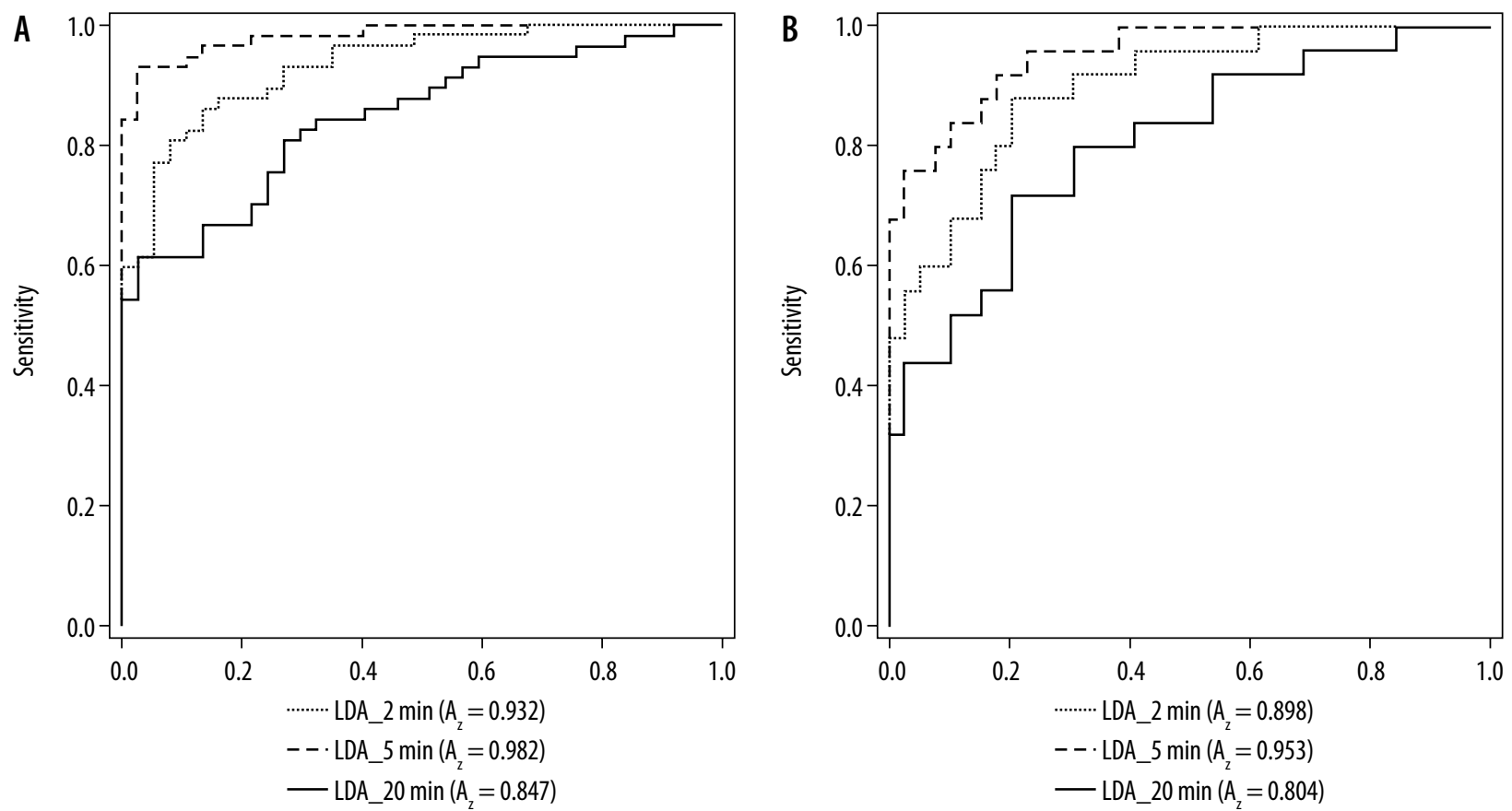

Figure 3. The diagrams of the receiver operating characteristic curve for texture analysis method with linear discriminant analysis (LDA) for classification of (A) rejected and non-rejected and (B) acute tubular necrosis and acute rejection in kidney transplant recipient. $A_{z}$ indicates area under the receiver operating characteristic curve

many classes of texture, and at least 19 of these texture features showed significant differences between rejected and non-rejected kidney transplants at each of the time frames. $A_{z}$ values of these texture features were determined in the range of 0.630 to 0.705 .

In general, according to the $\mathrm{A}_{\mathrm{z}}$-value, the co-occurrence matrix features had higher potential compared to other feature groups in terms of differentiation between rejected and non-rejected kidney transplants at the $2^{\text {nd }}$ and $5^{\text {th }}$ minute frames; the best classification of accuracy was achieved at the $5^{\text {th }}$ min frame. TA also showed good results in monitoring and determining GFR at each of the times. In this regard, the best result was achieved at the $20^{\text {th }}$ min frame, while at other frame times GFR had lower correlation coefficients.

$A_{z}$ values ordered by LDA using all texture features had a higher level of performance than did each of the texture features alone, according to classification of rejected and non-rejected groups. In this regard, $A_{z}$-value of LDA at $2^{\text {nd }}, 5^{\text {th }}$, and $20^{\text {th }}$ frame times were $0.932,0.982$, and 0.847 , respectively, while the highest level of performance was achieved by WavEnLH_s-6, correlate S $(0,1)$, and Teta2 with $A_{z}$-values of $0.693,0.705$, and 0.674 , respectively. Hence MPTA increased the information provided by scintigraphic imaging.

Since evaluations of mean age, BMI, and gender between the rejected and non-rejected kidney transplant recipients were determined as not significantly different, these parameters were not determined as confounding factors in this study.

At each frame time, among all texture features determined as significantly different between rejected and non-rejected groups, some texture features had no significant correlation with sCr level (or GFR). Based on a study by Kee et al., there is a subgroup of subclinical rejection defined as when histologic changes of AR were observed in the absence of an increased serum creatinine concentration [32]. From this reference it seems that pathological evidence of rejection in renal allograft had no correlation with the serum creatinine test. In addition, GFR was considered as a better criterion for monitoring kidney function and was advantageous over the $\mathrm{sCr}$ level because some GFR showed significant correlation with texture features while sCr levels did not show significant correlation.

AR and ATN are two common causes of impaired kidney function in the early post-transplantation period. Since scan patterns of severe ATN and AR are similar, perfusion indices cannot be considered as a good marker to distinguish them. Hence, biopsy remains the main method used for diagnosis [3,33-35]. Our results indicate that TA of scintigraphic imaging can provide additional information and has the potential to help physicians differentiate AR from ATN with an $\mathrm{A}_{\mathrm{z}}$-value of 0.953 .

Over the past few decades, quantitative studies have been employed to evaluate kidney function. Although ultrasound is often the primary choice for an initial assessment, follow-up, and monitoring of kidney transplants, scintigraphy has an advantage and can provide better information on kidney function $[13-15,17,18]$. In addition, according to research cited in the literature, there is no agreement on the resistance index (RI). Some studies have demonstrated that RI was a reliable marker to assess kidney function [12-14], but others have shown the opposite $[11,16-18]$. Hence, more research needs to be done 
to test RI before it can be recommended for use in clinical centres.

Many studies have indicated that MRI can detect changes in kidney function by using dynamic contrast enhanced MRI-derived values [25], diffusion-weighted imaging (DWI), and diffusion tensor imaging (DTI) [26-28]. Reports have shown $\mathrm{A}_{\mathrm{z}}$-values ranging from 0.865 to 0.885 , while our results showed a superior $\mathrm{A}_{\mathrm{z}}$-value at 0.982. Also, gamma cameras are more accessible in daily clinical practice, while DTI and DWI are more expensive and are less readily available. CT findings indicate that renal volumetry and perfusion measurements of kidney post-transplant have some correlation or even superior correlation to nuclear renography [19-22]. However, renal scintigraphy remains the referencing technique in clinical practice [6], although CT volumetry is sensitive to the degree of contrast agent injection. In addition, use of a contrast agent is limited in order to minimise the risk of toxic complications from iodinated and gadolinium-based intravenous contrast agents.

Relatively few studies have employed positron emission tomography (PET) imaging to evaluate kidney function, and the findings have been quite controversial. In this regard, mean standardised uptake values $\left(\mathrm{SUV}_{\text {mean }}\right)$ cannot be correlated with GFR, and a non-significant difference was determined between the kidney function groups tested in this study [23]. In contrast, $\mathrm{SUV}_{\text {mean }}$ had the potential to detect AR with sensitivity of $100 \%$, specificity of 50\%, and $A_{z}$-value of 0.930 [24]. The results of this study indicate that scintigraphic imaging, which is conventionally used in clinical centres, is more reliable than unconventional and expensive PET imaging.

In our previous study TA was employed to diagnose chronic kidney allograft rejection in ultrasound imaging, and the study indicated that TA had good potential for diagnosis of acute kidney allograft rejection [7]. Hence these results indicated that TA was a noninvasive, fast, and powerful technique for monitoring kidney function after transplantation.

The ability to detect and monitor kidney function during the post-transplantation period is an important factor for improving initial diagnoses. Further study using a larger dataset is needed to confirm these results. Scintigraphic classification based on TA was compared with pathology test results. Kidney function was also evaluated using sCr level. Because sCr level can be influenced by muscle mass, sex, age, and medication [36,37], cystatin-C indices may be a better reflection of the true GFR $[38,39]$, and definitive results require comparison with pathologic analysis (biopsy) and cystatin-C.

Becuase patient referrals were made on the basis of clinical indications, other kidney characteristics had no additional information and were not considered for TA. This method is not suggested as an alternative to a biopsy or serum examination, but it can be applied to help physicians identify subtle changes and select patients for biopsy that is classified as having a high risk of graft rejection.

\section{Conclusions}

In conclusion, we propose a new approach based on MPTA to evaluate the usefulness of renal graft scintigraphy for predicting renal function and to determine the best time point for this purpose. The best results were achieved at the $5^{\text {th }}$ and $20^{\text {th }}$ min frames to classify rejected and non-rejected kidney transplants and monitor $\mathrm{sCr}$ level, respectively. Also, MTA of scintigraphic imaging can provide additional information and has the potential to help physicians differentiate AR from ATN.

\section{Ethical disclosure}

All reported investigations were conducted in accordance with the principles of the Declaration of Helsinki and with our national regulations. All patients gave written, informed consent to use their data for research. This study was approved by the Ethics Committee of Iran University of Medical Sciences (no. IR.IUMS.REC 1395.95-04-3029953).

\section{Funding dources}

This work was supported by a grant (no. 29953) from Iran University of Medical Sciences (IUMS).

\section{Disclosure}

The authors declare no conflict of interests.

\section{References}

1. National Chronic Kidney Disease Fact Sheet: general information and national estimates on chronic kidney disease in the United States, 2010

2. Matas AJ, Smith JM, Skeans MA, et al. OPTN/SRTR 2011 Annual Data Report: Kidney. American Journal of Transplantation 2013; 13: 11-46.

3. Aktaş A. Transplanted kidney function evaluation. Semin Nucl Med 2014; 44: 129-145.
4. Dimitroulis D, Bokos J, Zavos G, et al. Vascular complications in renal transplantation: a single-center experience in 1367 renal transplantations and review of the literature. Transplant Proc 2009; 41: 1609-1614.

5. Danovitch GM (ed.). Handbook of Kidney Transplantation. Wolters Kluwer Health 2010.

6. Grenier N, Merville P, Combe C. Radiologic imaging of the renal parenchyma structure and function. Nat Rev Nephrol 2016; 12: 348-359. 
7. Abbasian Ardakani A, Mohammadi A, Khalili Najafabad B, Abolghasemi J. Assessment of Kidney Function After Allograft Transplantation by Texture Analysis. Iran J Kidney Dis 2017; 11: 157-164.

8. Materka A. Texture analysis methodologies for magnetic resonance imaging. Dialogues Clin Neurosci 2004; 6: 243-250.

9. Castellano G, Bonilha L, Li LM, Cendes F. Texture analysis of medical images. Clin Radiol 2004; 59: 1061-1069.

10. Ardakani AA, Gharbali A, Saniei Y, et al. Application of Texture Analysis in Diagnosis of Multiple Sclerosis by Magnetic Resonance Imaging. Global J Health Sci 2015; 7: 68-78.

11. Bou Matar R, Warshaw B, Hymes L, et al. Routine transplant Doppler ultrasonography following pediatric kidney transplant. Pediatr Transplant 2012; 16: 607-612.

12. Cano H, Castañeda DA, Patiño N, et al. Resistance index measured by Doppler ultrasound as a predictor of graft function after kidney transplantation. Transplant Proc 2014; 46: 2972-2974.

13. Patel K, Patel N, Gandhi S. Comparison between doppler ultrasound resistive index, serum creatinine, and histopathologic changes in patients with kidney transplant dysfunction in early posttransplantation period: A single center study with review of literature. Saudi J Kidney Dis Transpl 2016; 27: 533-538.

14. Yazici B, Yazici A, Oral A, et al. Comparison of renal transplant scintigraphy with renal resistance index for prediction of early graft dysfunction and evaluation of acute tubular necrosis and acute rejection. Clin Nucl Med 2013; 38: 931-935.

15. Lee J, Oh YT, Joo DJ, et al. Acoustic Radiation Force Impulse Measurement in Renal Transplantation: A Prospective, Longitudinal Study With Protocol Biopsies. Medicine (Baltimore) 2015; 94: e1590.

16. Gao J, Rubin JM, Weitzel W, et al. Comparison of Ultrasound Corticomedullary Strain with Doppler Parameters in Assessment of Renal Allograft Interstitial Fibrosis/Tubular Atrophy. Ultrasound Med Biol 2015; 41: 2631-2639.

17. Yazici B, Oral A, Gokalp C, et al. Evaluation of renal transplant scintigraphy and resistance index performed within 2 days after transplantation in predicting long-term graft function. Clin Nucl Med 2015; 40: 548-552.

18. Yoon Y-C, Shin BS, Ohm JY, et al. Comparison between Doppler Ultrasonography and Renal Scintigraphy in Assessment of Post-Transplant Renal Function. J Korean Soc Radiol 2016; 74: 313-321.

19. Gaillard F, Pavlov P, Tissier A-M, et al. Use of computed tomography assessed kidney length to predict split renal GFR in living kidney donors. Eur Radiol 2017; 27: 651-659.

20. Barbas AS, Li Y, Zair M, et al. CT volumetry is superior to nuclear renography for prediction of residual kidney function in living donors. Clin Transplant 2016; 30: 1028-1035.

21. Yanishi M, Kinoshita H, Yoshida T, et al. Comparison of renal scintigraphy and computed tomographic renal volumetry for determining split renal function and estimating post-transplant renal function. Transplant Proc 2015; 47: 2700-2702.

22. Patankar K, Low R, Blakeway D, et al. Comparison of computer tomographic volumetry versus nuclear split renal function to determine residual renal function after living kidney donation. Acta Radiol 2014; 55: 753-760.

23. Jadoul A, Lovinfosse P, Weekers L, et al. The Uptake of 18F-FDG by renal allograft in kidney transplant recipients is not influenced by renal function. Clin Nucl Med 2016; 41: 683-687.
24. Lovinfosse P, Weekers L, Bonvoisin C, et al. Fluorodeoxyglucose F18 Positron Emission Tomography Coupled With Computed Tomography in Suspected Acute Renal Allograft Rejection. Am J Transplant 2016; 16: 310-316

25. Eikefjord E, Andersen E, Hodneland E, et al. Quantification of Single-Kidney Function and Volume in Living Kidney Donors Using Dynamic Contrast-Enhanced MRI. AJR Am J Roentgenol 2016; 207: 1022-1030.

26. Palmucci S, Cappello G, Attinà G, et al. Diffusion weighted imaging and diffusion tensor imaging in the evaluation of transplanted kidneys. Eur J Radiol 2015; 2: 71-80.

27. Ren T, Wen CL, Chen LH, et al. Evaluation of renal allografts function early after transplantation using intravoxel incoherent motion and arterial spin labeling MRI. Magn Reson Imaging 2016; 34 : 908-914.

28. Hueper K, Khalifa A, Bräsen J, et al. Diffusion-weighted imaging and diffusion tensor imaging detect delayed graft function and correlate with allograft fibrosis in patients early after kidney transplantation. J Magn Reson Imaging 2016; 44: 112-121.

29. ACR-SPR practice parameter for the performance of renal scintigraphy. Available at: https://www.acr.org/Quality-Safety/Standards-Guidelines/Practice-Guidelines-by-Modality/Nuclear-Medicine.

30. Cockcroft DW, Gault MH. Prediction of creatinine clearance from serum creatinine. Nephron 1976; 16: 31-41.

31. Van Erkel AR, Pattynama PMT. Receiver operating characteristic (ROC) analysis: basic principles and applications in radiology. Eur J Radiol 1998; 27: 88-94.

32. Kee TY, Chapman JR, O'Connell PJ, et al. Treatment of subclinical rejection diagnosed by protocol biopsy of kidney transplants. Transplantation 2006; 82: 36-42.

33. Sharfuddin A. Imaging evaluation of kidney transplant recipients. Semin Nephrol 2011; 31: 259-271.

34. Aktas A, Karakayali H, Bilgin N, et al. Serial radionuclide imaging in acute renal allograft dysfunction. Transplant Proc 2002; 34: 21022105.

35. Gupta S, Lewis G, Rogers K, et al. Quantitative 99mTc DTPA renal transplant scintigraphic parameters: assessment of interobserver agreement and correlation with graft pathologies. Am J Nucl Med Mol Imaging 2014; 4: 213-224.

36. van Acker BAC, Koopman MG, Arisz L, et al. Creatinine clearance during cimetidine administration for measurement of glomerular filtration rate. Lancet 1992; 340: 1326-1329.

37. Andreev E, Koopman M, Arisz L. A rise in plasma creatinine that is not a sign of renal failure: which drugs can be responsible? J Intern Med 1999; 246: 247-252.

38. Randers E, Erlandsen EJ. Serum cystatin C as an endogenous marker of the renal function - a review. Clin Chem Lab Med 1999; 37: 389-395.

39. Gökkuşu CA, Özden TA, Gül H, et al. Relationship between plasma Cystatin $\mathrm{C}$ and creatinine in chronic renal diseases and Tx-transplant patients. Clin Biochem 2004; 37: 94-97. 